\title{
Perspective
}

PERSPECTIVE Actualité en histoire de l'art

2| 2015

Les États-Unis

\section{Des objets paradoxaux : les quilts dans la culture américaine}

Janneken Smucker

Traducteur : Géraldine Bretault

(2) OpenEdition

Journals

Édition électronique

URL : http://journals.openedition.org/perspective/6173

DOI : $10.4000 /$ perspective. 6173

ISSN : 2269-7721

Éditeur

Institut national d'histoire de l'art

Édition imprimée

Date de publication : 31 décembre 2015

Pagination : 155-162

ISBN : 978-2-917902-27-1

ISSN : $1777-7852$

\section{Référence électronique}

Janneken Smucker, «Des objets paradoxaux : les quilts dans la culture américaine », Perspective [En ligne], 2 | 2015, mis en ligne le 30 juin 2017, consulté le 01 octobre 2020. URL : http://

journals.openedition.org/perspective/6173; DOI : https://doi.org/10.4000/perspective.6173 


\section{Des objets paradoxaux : les quilts dans la culture américaine}

\author{
Janneken Smucker
}

D'après une étude de 2014 , les États-Unis comptent 16,4 millions de confectionneurs de quilts (courtepointes), dans une industrie du quilt évaluée à 3,76 milliards de dollars ${ }^{1}$. Comme au cours des deux siècles précédents, le quilting (art de confectionner un quilt) demeure un support d'expression artistique, ainsi qu'un artisanat faisant appel à un véritable savoir-faire et une grande dextérité technique. À l'instar de leurs prédécesseurs, les confectionneurs de quilts contemporains, apprécient le charme de cet artisanat en apparence désuet, mais qu'ils pratiquent en utilisant des machines à piquer, des machines à découpe, et à l'aide de tutoriels vidéo. Ils confectionnent des quilts chez eux - pour les utiliser comme couvre-lits et décoration murale -, tout en participant à tous les aspects de l'industrie de masse du quilt : ils assistent à des foires, suivent les célébrités du quilt sur les réseaux sociaux, collectent des chutes de tissus et achètent de nouveaux accessoires. Certains confectionneurs contemporains de quilts cherchent à produire des quilts en patchwork à l'aspect rapiécé (scrappy), évoquant le fait-main, en dépit d'une réalité démographique établie de longue date, à savoir que les confectionneurs de quilts sont plutôt aisés. À l'heure actuelle, ils comptent parmi eux des artistes produisant des pièces originales et techniques, ainsi que des amateurs produisant des quilts conventionnels. Nombre d'entre eux se tournent vers la communauté pour travailler selon cette coutume ancienne, tout en fabriquant des quilts individuels fondés sur l'expression personnelle.

Ces tendances ne sont pas nouvelles. L'art du quilt aux États-Unis a longtemps été caractérisé par des tensions culturelles, entre art et artisanat, ancien et moderne, production domestique mais dépendante de l'industrialisation, évoquant la pauvreté alors qu'il est lié à l'abondance, avantgardiste mais conventionnel et tourné vers la communauté tout en reflétant une créativité individuelle. Comme pour les générations précédentes, les confectionneurs de quilts actuels continuent de repousser les limites de ce médium, brouillant les frontières qui définissent ces paradoxes.

Le quilt American Gothic de l'artiste Luke Haynes aborde un certain nombre de ces contradictions. La qualité très picturale de son portrait, ainsi que le titre et la composition elle-même, évoquent le régionalisme américain ${ }^{2}$, bien que l'aspect artisanal du fond en patchwork situe la pièce parmi les quilts traditionnels. Haynes recycle de vieux vêtements, puisant avec nostalgie dans un passé fantasmé où les confectionneurs de quilts devaient faire avec ce qu'ils avaient "sous la main ", alors que lui-même s'approvisionne dans des friperies regorgeant de vieux vêtements. Des quilts comme celui-ci nous permettent d'étudier les puissantes motivations humaines qui se cachent derrière ces créations : la fantaisie, le progrès, la nostalgie, l'individualisme et la collectivité (fig. 1).

\section{Art/Artisanat}

Un paradoxe essentiel du quilting tient à sa position face à la frontière bien gardée qui sépare l'art de l'artisanat. Les débats sur ces catégories hiérarchiques échouent à produire des étiquettes définitives, étant donné que les quilts se tiennent en équilibre instable entre l'art, le design et l'artisanat ${ }^{3}$. Cependant, à plusieurs reprises au cours du siècle passé, l'exclusion ou l'inclusion des quilts dans la catégorie " art " a révélé à la fois le contexte plus large de la césure entre art et artisanat au sein de la société, ainsi que le rôle spécifique des quilts.

Depuis le $\mathrm{XIX}^{\mathrm{e}}$ siècle, les femmes considèrent leurs quilts comme de l'art. Elles puisent leur inspiration dans d'autres domaines artistiques et

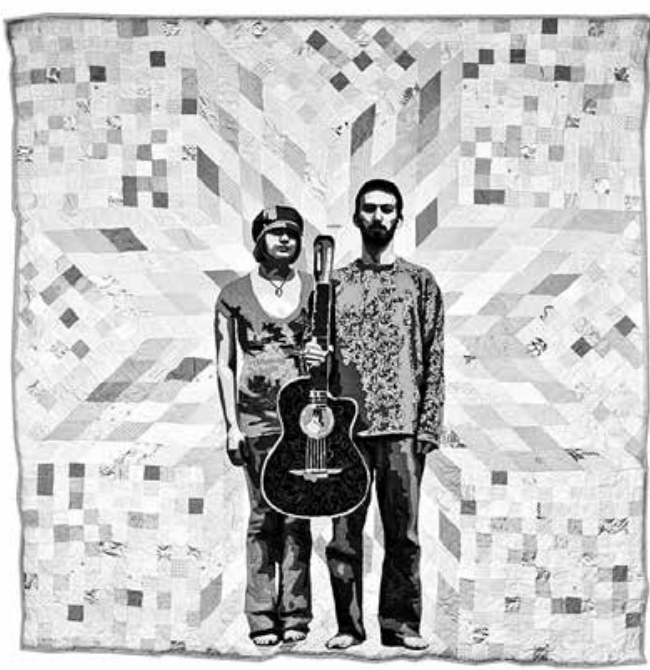

1. Luke Haynes, American Gothic, American Context $\# 3,2010$. 
exposent publiquement leur production dans un certain nombre de foires et de collectes de fonds ${ }^{4}$. Toutefois, les Américains ont rarement considéré les quilts dans un contexte artistique, avant que l'influence du mouvement Arts and Crafts et du modernisme ne se fasse sentir sur les arts décoratifs américains, à partir du début du $\mathrm{XX}^{\mathrm{e}}$ siècle. Des artistes femmes et des créatrices, formées professionnellement, diplômées de nouvelles écoles d'art, se sont mises à créer des motifs de quilts modernistes. En outre, les collectionneurs d'objets d'art anciens américains ont commencé à établir des liens entre les quilts anciens et l'art moderniste, tandis que les modernistes se tournaient vers les arts populaires américains envisagés comme un héritage artistique pour leur travail contemporain ${ }^{5}$.

Lorsque les amateurs d'art newyorkais ont " découvert " les quilts, à la fin des années 1960 et dans les années 1970, ils les ont comparés aux œuvres d'art abstrait et les ont accrochés à leurs murs. Les quilts se sont imposés dans les débats sur la hiérarchie entre art et artisanat ${ }^{6}$. Au moment même où les beaux-arts embrassaient le Pop Art, l'art textile et l'art conceptuel, les quilts se sont immiscés dans la conversation. Certains artistes contemporains en se consacrant au quilt, en ont fait leur médium principal, en donnant naissance au mouvement du "Studio Art Quilt " ${ }^{7}$.

Pourtant, la tension entre art et artisanat ne s'est pas résorbée. Alors que certains conservateurs et collectionneurs militaient pour que les quilts soient appréciés comme des œuvres d'art, les tenants d'une distinction entre art et artisanat refusaient de capituler. En 1975, la veuve du peintre expressionniste abstrait Barnett Newman, Annalee, a opposé les tableaux de son défunt mari aux quilts, en déclarant que Newman cherchait à explorer les « relations subtiles entre les rayures et le fond ", tandis qu'un confectionneur de quilt se contente "d'exécuter un motif simple " 8 . Plus d'une décennie plus tard, le critique d'art Robert Hughes observait à propos des quilts des Amish : "Pris hors de leur contexte d'origine, accrochés à un mur, ils démontrent l'absurdité des distinctions hiérarchiques autrefois farouches entre art 'majeur' et 'art populaire' $"$ " . Comme l'illustre cette déclaration de Hughes, si l'on veut considérer les quilts traditionnels qui servaient de courtepointes comme des ouvres d'art, il faut d'abord les décontextualiser. Ou, comme l'a noté la spécialiste Susan Bernick : «Un quilt est un objet d'art lorsqu'il se tient droit comme un homme ${ }^{10}$.

De fait, la classification des quilts comme relevant de l'art ou de l'artisanat s'est inscrite dans le débat plus large lié au gender studies. Dans les années 1970, tandis que des hommes, collectionneurs, et des conservateurs accrochaient des quilts à leurs murs et écrivaient à propos de la puissance visuelle de ces quilts, des chercheuses féministes tentaient de les re-contextualiser, en rappelant qu'ils étaient les produits des mains habiles de femmes, intimement liés aux divers univers féminins, et pas seulement de beaux objets décoratifs ${ }^{11}$. En examinant les quilts dans leurs contextes variés, nous pouvons échapper à cette dichotomie simpliste entre art et artisanat pour atteindre une meilleure compréhension de ces objets, à partir d'une multitude de perspectives.

\section{Ancien/Moderne}

La conviction que le quilting est une tradition, c'est-à-dire qu'il renvoie à un mode de vie ancien, n'a jamais faibli aux États-Unis, en dépit de la modernisation constante de cet artisanat. À l'époque coloniale, seuls les Américains les plus aisés possédaient des quilts, car les textiles étaient importés d'Europe à des prix élevés. Les progrès industriels des années 1820-1840 ont démocratisé l'art du quilt, grâce à une hausse de l'approvisionnement en cotonnades, assortie d'une baisse des prix. Cependant, à partir des années 1840, certains Américains portèrent un regard nostalgique sur les quilts, comme s'ils étaient les reliques d'une époque révolue, même leur production faisait appel à des matériaux, des motifs et des technologies inédits ${ }^{12}$.

À la fin du XIX ${ }^{\mathrm{e}}$ siècle et au début du $\mathrm{XX}^{\mathrm{e}}$ siècle, les quilts demeuraient étroitement associés avec un passé rêvé, dans le contexte du "Colonial Revival ", attaché à célébrer tout ce qui est ancien, et pouvant être relié de manière mythique ou réelle à des temps plus simples, antérieurs à l'industrialisation et l'urbanisation. Les tenants du Colonial Revival ont réinterprété les motifs de quilts autrefois populaires auprès des générations antérieures; ils s'habillaient avec des vêtements coloniaux et se réunissaient lors d'ateliers de quilting; ils associaient des quilts modernes du XIX ${ }^{\mathrm{e}}$ siècle avec des parures de lits $\mathrm{d}^{\prime}$ inspiration coloniale ${ }^{13}$. 
En dépit de ces liens avec un passé imaginaire, les confectionneurs de quilts - ainsi que les partisans de l'industrie du quilt - ont tout de même fini par adapter leur production aux temps modernes. Grâce à des techniques, des motifs et des méthodes inédits - incluant la machine à coudre, les kits de quilting et le cutter rotatif, désormais omniprésent -, ces quilts pouvaient évoquer le meilleur de l'ancien temps, en dépit de leur exécution moderne ${ }^{14}$.

Bien que la fabrication des quilts ait persisté pendant la Seconde Guerre mondiale, une seconde renaissance s'est imposée au début des années 1970, qui ne s'est pas démentie jusqu'en ce début de XXI siècle. La culture actuelle du quilt a entretenu une vision nostalgique du quilting, tout en relançant le secteur grâce à des nouvelles technologies, ainsi qu'un accès facilité aux outils, aux tissus et aux instructions, grâce à Internet. De nos jours, le quilt américain est aussi moins distinctement américain, dans la mesure où la mondialisation a favorisé les échanges : l'art du quilt à l'américaine a traversé l'Atlantique en sens inverse, pour gagner ensuite l'Asie, l'Amérique latine et le reste du monde ${ }^{15}$.

\section{Domestique/Industriel}

Un autre mythe persistant considère les quilts comme une forme de production exclusivement domestique et féminine. Marie Webster, créatrice de quilts et auteure de l'ouvrage Quilts: Their Story and How to Make Them (1915), louait les talents des femmes de l'époque coloniale en matière de quilting, alors même que peu de femmes à cette époque produisaient des quilts dans un contexte domestique, en raison du prix élevé des textiles issus de l'importation ${ }^{16}$.

Dans les années 1970, les interprétations féministes ont continué à mettre en avant l'art du quilt comme une forme de domesticité féminine et comme une tradition par essence féminine, qualifiée "d'art incontestablement féminin ${ }^{17}$. Les féministes voyaient dans les travaux d'aiguille de ces femmes les états précurseurs des formes émergentes d'une pratique artistique féministe. De grandes artistes féministes comme Judy Chicago, Faith Ringgold et Miriam Shapiro ont intégré des techniques de couture et des formes d'art liées au quilting dans leur travail, allant à l'encontre d'une stricte distinction entre arts majeurs et arts appliqués, ces derniers étant souvent relégués aux débats sur le genre ${ }^{18}$. Ce faisant, en insistant sur la valeur de patrimoine artistique féminin du quilt, elles ont négligé les liens étroits associant le quilting à l'industrialisation et au commerce.

Lorsque des fabriques ont vu le jour en Nouvelle-Angleterre, le quilting est devenu un produit de l'industrialisation autant que de la sphère domestique, grâce aux tissus de production industrielle, aux fils fabriqués par des machines, aux aiguilles et aux broches confectionnées à la chaîne, et aux teintures à l'aniline, sans oublier la machine à coudre ${ }^{19}$. Outre les matériaux et les machines destinés à produire des quilts, l'industrialisation a engendré les infrastructures indispensables pour publier les motifs, assurer une distribution élargie du matériel nécessaire et soutenir les petites et grandes entreprises de quilt, assimilant le quilting à un art accessible et démocratique ${ }^{20}$.

Dans les faits, depuis le XIX ${ }^{e}$ siècle, les femmes pratiquant le quilting au foyer ont produit la vaste majorité des quilts, mais elles se sont souvent tournées vers le monde extérieur pour y puiser leur inspiration. À l'heure actuelle, les célébrités du quilt stimulent la créativité des confectionneurs amateurs de quilts. Le même phénomène s'était déjà produit au début du XIX ${ }^{\mathrm{e}}$ siècle, lorsque des célébrités du quilting - réelles comme Marie Webster, Ann Orr et Ruby Short McKim, imaginaires, comme les personnages de fiction Nancy Page et Nancy Cabot - se sont mises à diffuser des conseils et des motifs de quilting dans les magazines féminins et dans les colonnes de journaux ${ }^{21}$. Ce phénomène de célébrité du quilt a peut-être un antécédent : des spécialistes émettent aujourd'hui l'hypothèse que certains des blocs d'impression ornés des quilts supérieurs appelés "Baltimore Albums » du milieu du XIX ${ }^{e}$ siècle étaient produits commercialement par au moins un fabricant à Baltimore, qui vendait probablement ces motifs ${ }^{22}$.

\section{Pauvreté/Abondance}

Les quilts américains incarnent l'idée-même d'une production créative faite à partir de trois fois rien, en cousant plusieurs morceaux de tissus ensemble. Pour décrire cette notion qui consiste à voir le quilt comme un produit lié à la pauvreté, les spécialistes font appel au "mythe du sac à chutes de tissus ", qui part du principe que les femmes des premiers temps des États-Unis fouillaient dans leur sac à chutes de tissus et recyclaient des vêtements et du linge de maison usés pour fabriquer des quilts 
2. Quilt d'un artiste inconnu, vers 1975-

2000, trouvé en Alabama, collection de Roderick Kiracofe.

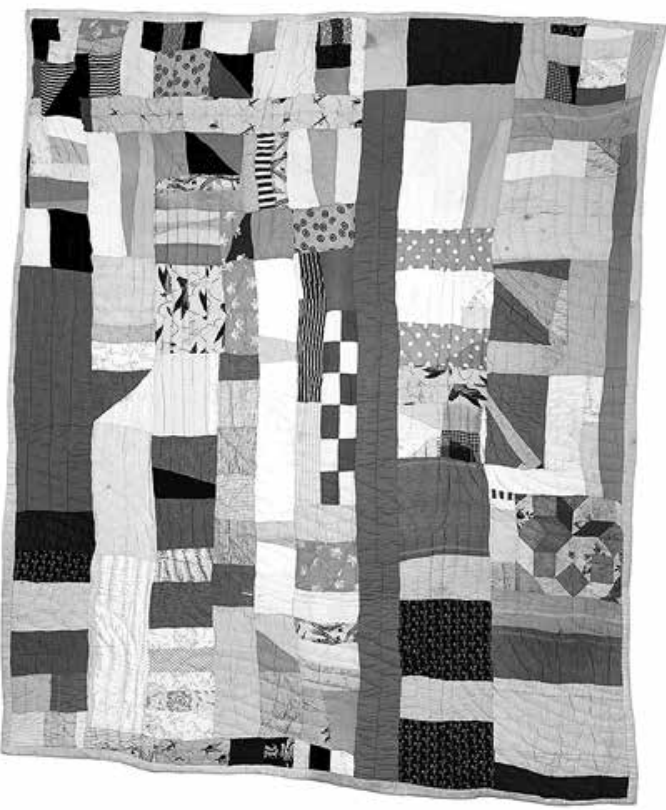

destinés à garder leur famille bien au chaud ${ }^{23}$. Si certains quilts sont le résultat d'un remploi inspiré, la grande majorité des quilts conservés des XVIII et XIX ${ }^{e}$ siècles étaient composés de tissus plutôt neufs qu'usés. La confection de quilts a explosé avec l'abondance introduite par la Révolution industrielle et l'émergence de la consommation de masse, qui ont permis aux femmes d'acheter du tissu rapidement pour confectionner des quilts, ou d'utiliser les chutes de tissu qui leur restaient après avoir cousu des vêtements ${ }^{24}$.

Les auteurs du Colonial Revival ont mis en avant le rôle du sac à chutes de tissus. En 1915, Marie Webster avançait l'hypothèse que, pour que les quilts maintiennent les colons au chaud durant les hivers rigoureux, " chaque morceau de tissu récupéré de la confection des vêtements était épargné. Pour compléter ceux-ci, les parties en meilleur état sur les vêtements usés étaient découpées, et transformées en pièces pour quilts $»^{25}$. En 1929, Ruth Finley a déclaré : « Dans les maisons de maitre comme dans les cabanons de la Frontière, des cavaliers de la Virginie aux puritains de Plymouth, les chutes de lin, de coton, de soie et de laine étaient jalousement gardées et rapiécées pour former des quilts en patchwork $»^{26}$.

En se basant sur les quilts conservés depuis les premiers temps de l'Amérique, ces premiers spécialistes des quilts ont imaginé que la pauvreté fondait la tradition du quilt. Mais lorsqu'est survenue la Grande Dépression, les femmes ont réellement été contraintes de remployer les vieux vêtements pour fabriquer des quilts. Un nombre conséquent de quilts du $\mathrm{XX}^{\mathrm{e}}$ siècle trahit ce souci économique, bien que leur reconnaissance en tant qu'objets d'art soit très récente, dans la mesure où les collectionneurs comme les conservateurs de quilts se sont longtemps intéressés davantage aux quilts " supérieurs " plutôt qu'aux plus rapiécés. L'exposition itinérante à succès, Quilts of Gee's Bend (2002-2006) présentait des quilts utilitaires réalisés à partir de chutes de tissu par des producteurs afroaméricains dans une enclave isolée de l'Alabama ${ }^{27}$. Plus récemment, l'ouvrage du collectionneur Roderick Kiracofe, Unconventional and Unexpected: American Quilts Under the Radar, 1950-2000, contenait quelques quilts rapiécés excentriques montrant que l'économie et le remploi sont des aspects récurrents de la confection de quilts au $\mathrm{XX}^{\mathrm{e}}$ siècle ${ }^{28}$. Les motifs joyeux et improvisés, ainsi que l'usage audacieux et graphique de tissus inattendus ont rendu ces quilts aussi attrayants que de l'art abstrait. Ils sont aussi devenus les symboles de l'ingéniosité créative des femmes, comblant enfin l'écart entre art et artisanat (fig. 2).

\section{Avant-garde/Convention}

De nombreux confectionneurs de quilts se sont contentés de produire des quilts semblables à ceux de leurs voisins, ou d'imiter les modèles présentés dans les magazines, quand ils n'utilisaient pas des kits ou des patrons publiés dans le commerce. Pour cette raison, les confectionneurs de quilts ont cousu des milliers de quilts avec des motifs appliqués rouges et verts durant les années 18501860, puis des "Crazy Quilts " dans les années 1870-1890, et enfin des quilts " Grandmother's Flower Garden » dans les années 1920-1930. Cependant, parmi ces styles conventionnels, des confectionneurs de quilts au talent remarquable ont exploité le tissu comme un support neutre pour créer des ouvres d'art originales.

Dans son Reconciliation Quilt, Lucinda Honstain a recours à l'appliqué pour dresser un récit de la vie quotidienne comme de faits imaginaires survenus pendant et après la guerre de Sécession à Brooklyn. Dans ses quarante cases, Honstain décrit des créatures exotiques, comme un chameau, un éléphant et un morse, ainsi qu'un vendeur d'articles de mercerie, des Afro-Américains libres et d'autres réduits en esclavage, ou encore des Zouaves inspirés des soldats français, vêtus 
de pantalons rouge vif et de vestes bleues ${ }^{29}$. À l'époque des "Crazy Quilts " (1880-1890), alors que les appliqués de soie et de velour étaient nombreux, Harriet Powers, une confectionneuse modeste de quilts afro-américaine, qui avait grandi comme esclave, créa de célèbres quilts à partir des histoires bibliques (fig.3). Elle vendit le premier à un client blanc, pour cinq dollars ${ }^{30}$. Les créateurs les plus audacieux ont soumis leurs quilts en 1933 au concours national de quilt organisé par Sears Roebuck \& Company, dans le cadre de l'Exposition universelle de Chicago, aussi connue sous le nom de "A Century of Progress ". Parmi les 24000 participants, certains avaient repris des motifs modernistes, inspirés notamment par l'architecture Art déco de Chicago et l'esthétique aérodynamique des années 1930, pour créer des quilts originaux. Par exemple, Emma Mae Leonhard créa un quilt retraçant l'évolution de la mode féminine entre 1833 et 1933, en y associant de l'architecture Art déco et des symboles patriotiques stylisés (fig. 4) ${ }^{31}$.

Les styles de certains quilts étaient relativement familiers à la communauté de leur créateur, mais ils étaient considérés comme des exemples de motifs exceptionnels du point de vue d'observateur étranger. Au cours de la première moitié du $\mathrm{XX}^{\mathrm{e}}$ siècle, les femmes des Amish de l'Ancien Ordre dans le comté de Lancaster, en Pennsylvanie, ont réalisé des centaines de quilts "Center Diamond ", dans des myriades de déclinaisons, basées sur la couleur; de subtiles différences dans les motifs des quilts et un usage varié des bordures, des cadres et des coins. Ces quilts présentent des similarités visuelles avec la série Hommage au carré de Josef Albers (1956, Paris, Centre Pompidou), qui explore la relation de la couleur avec une formule géométrique rigide. De la même manière, beaucoup de femmes issues des milieux ruraux pauvres fabriquaient des quilts improvisés, conventionnels au sein de leurs communautés, mais considérés aujourd'hui comme de grandes œuvres d'art graphique (fig. 5).

\section{Communauté/Individu}

Le quilting bee (atelier de quilting) - dont le souvenir réapparaît dans les années 1840, tout en étant figuré dans l'art aux XIX et XX $X^{\mathrm{e}}$ siècles et pratiqué à nouveau par les nostalgiques des temps anciens - suggère l'importance de la communauté dans l'art du quilt. Les quilting bees ou quilting frolics comme on appelait autrefois ces rassemblements, étaient des événements importants sur le plan social, au cours desquels des amies et des parentes se réunissaient autour d'un cadre de quilting et cousaient à la main les couches de tissu constituant le quilt - une tâche laborieuse, qui aurait représenté d'innombrables heures de travail en solitaire. Ce travail collectif est resté habituel parmi différentes communautés de confectionneurs de quilts, dont les Amish, les Afro-américains, les Amérindiens et différents ateliers de couture de paroisse. Vers la fin du $\mathrm{XX}^{\mathrm{e}}$ siècle, des anonymes proches se sont rassemblés pour célébrer à travers leurs quilts leurs proches disparus, dans le cadre du " AIDS Memorial Quilt ». Ce projet populaire fondé en 1987 regroupe aujourd'hui 48000 panneaux individuels en hommage aux victimes du HIV et du SIDA ${ }^{32}$. Cet aspect collectif de l'art du quilt comme facteur de solidarité communautaire a entraîné l'adoption générale du quilt par divers groupes cherchant à préserver cette tradition à une époque de mutation rapide.

Plusieurs groupes se sont servis en effet du quilt pour resserrer de manière symbolique les liens entre les membres de leur communauté. $\mathrm{Au} \mathrm{XIX}{ }^{\mathrm{e}}$ siècle, les églises ont fabriqué des quilts qui étaient expédiés aux pasteurs lorsque ceuxci quittaient la communauté pour une autre paroisse. Les proches signaient de leur nom des

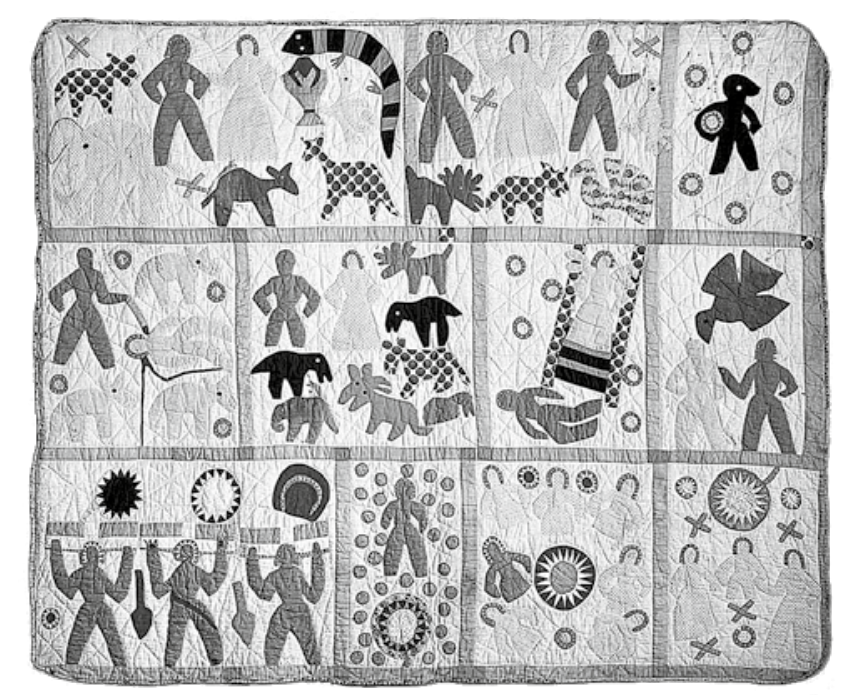

3. Harriet Powers, Bible Quilt, 18851886, Washington, D.C., National Museum of American History. 
4. Emma Mae Leonhard, De 1833 à 1933, Virginie (Illinois), vers 1933, Lincoln, University of NebraskeLincoln, International Quilt Study Center \& Museum.

5. Quilt créé par un artisan Amish inconnu dans le comté de Lancaster (Pennsylvanie), vers 19201940, Lincoln, University of NebraskeLincoln, International Quilt Study Center \& Museum.

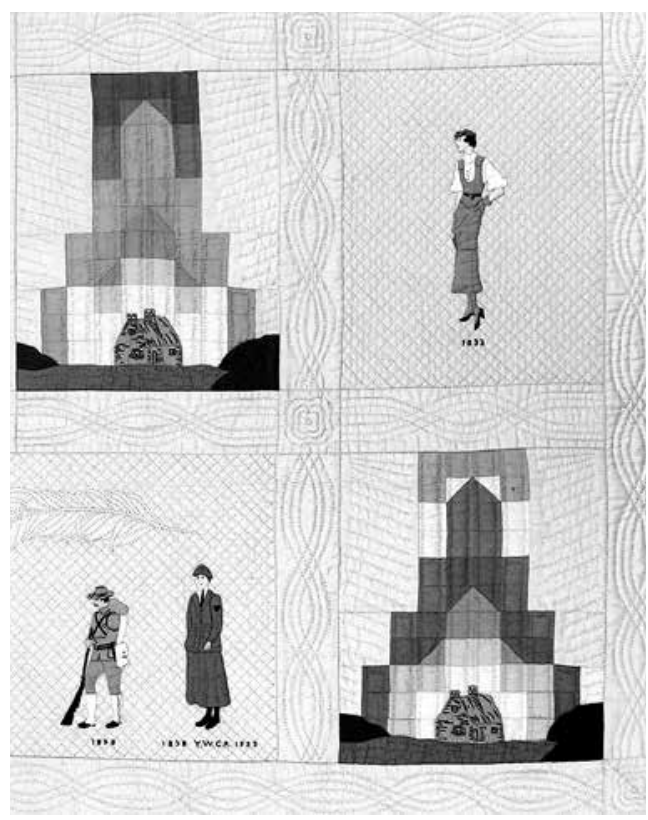

entreprise créative dans laquelle une personne choisit des tissus, sélectionne un motif ou invente un dessin, et travaille seul. Les confectionneurs de quilts prennent des décisions individuelles petites et grandes - qui débouchent sur des quilts originaux. Pour de nombreuses femmes ayant peu d'autres débouchés créatifs, les quilts ont représenté une forme d'expression personnelle. Mary Hernandred Ricard a travaillé pendant trente-cinq ans, jusqu'à plus de soixante-dix ans, sur un quilt qu'elle a baptisé My Crazy Dream, suggérant qu'elle avait puisé son inspiration à partir d'une vision personnelle. Elle l'a inscrite et datée, et brodé tout un répertoire iconographique fantastique, incluant des animaux exotiques et des scènes tirées des contes de fées. Par ailleurs, elle a intégré sa propre photographie d'identité, pour bien montrer que son quilt était une forme d'autoreprésentation ${ }^{34}$.

\section{L'attrait persistant des quilts américains}

" Friendship Quilts » qui étaient offerts à ceux qui émigraient vers l'Ouest ou qui allaient se marier. Les mères Amish produisaient des quilts qu'elles offraient comme " cadeaux de la maison » à leurs enfants lorsqu'ils quittaient le foyer pour fonder leurs propres familles. Les membres des tribus amérindiennes offraient des "Star Quilts " comme présents honorifiques. À travers ces exemples, on constate que le caractère utilitaire des quilts a disparu devant leur fonction symbolique ${ }^{33}$.

Aux antipodes de cette fonction protectrice du quilt envers une communauté donnée, la confection de quilts est aussi un acte solitaire, une

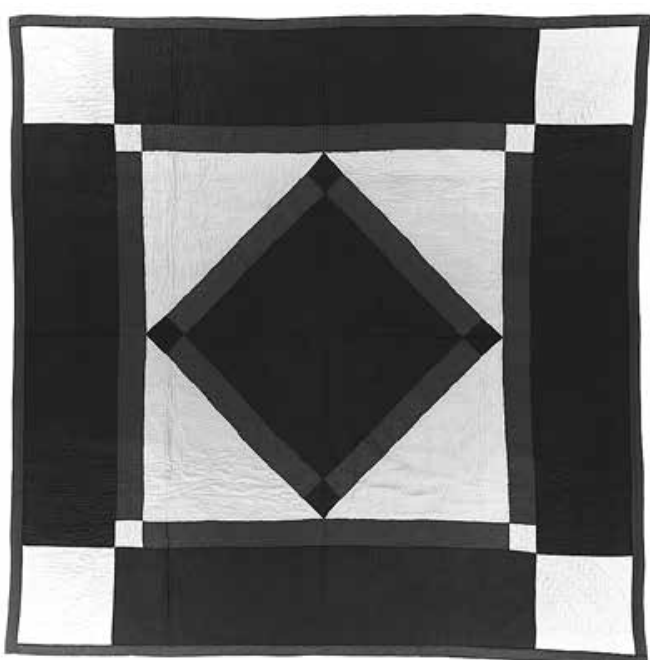

Pourquoi est-ce que pendant des siècles des femmes américaines - et des hommes, de plus en plus -, se sont adonnés au quilting ? Bien que les motivations individuelles soient très variables, ces quilts n'ont jamais été envisagés comme un moyen pratique de confectionner un couvre-lit. Découper des morceaux de tissu pour les coudre ensuite ensemble est un acte plutôt risible. Était-ce pour la créativité requise et l'impression de se donner un but qui en découle ? Peut-être faut-il voir dans cette formidable attraction pour les quilts de la part d'un si large éventail de communautés et d'individus une forme d'expression individuelle, que l'on trouve dans My Crazy Dream de Ricard comme dans American Gothic de Luke Haynes. Tous deux ont d'ailleurs inclus dans ces quilts la figure de l'artiste. Pour bien des individus créatifs, la confection de quilts a représenté un débouché accessible et sans danger pour ce type d'expression, dans la mesure où les qualités durables des quilts en tant qu'objets doux, agréables au toucher, véhiculant chaleur et confort rendent cet art - ou cet artisanat ? - attrayant auprès de tous ceux qui ont du temps, du tissu et des compétences en couture.

À l'image des tensions énumérées ci-dessus, les quilts réunissent bien des contradictions qui demeurent présentes au sein de la société américaine. C'est peut-être la raison pour laquelle les quilts ont si souvent servi de métaphore pour 
tous les aspects de la société faisant appel à la créativité, au courage, etc. Les quilts, dans toute leur complexité, continuent de résonner dans la culture américaine, et aujourd'hui peut-être plus que jamais.

\section{Cet article a été traduit par Géraldine Bretault.}

1. F+W, A Content + eCommerce Company, "Quilting in America (TM) 2014 ", dans Quilting in America, 2014.

2. Le régionalisme américain est un mouvement artistique américain prônant le retour aux valeurs rurales et nationales, en vogue dans les années 1930 (N.D.L.T.).

3. Perspectives: Art, Craft, Design $\theta$ the Studio Quilt, Michael James, Sandra Sider, (cat. expo., Lincoln, University of Nebraska, International Quilt Study Center \& Museum, 2009-2010), Lincoln, 2009 ; Christine Humphery, "Quilts as Art ", dans World Quilts: The American Story | International Quilt Study Center $\theta$ Museum, 2013, http://worldquilts.quiltstudy.org/americanstory/creativity/quiltsasart (consulté le 30 novembre 2015).

4. Elaine Hedges, Julie Silber, Pat Ferrero, Hearts and Hands: Women, Quilts, and American Society, Nashville, 1996, p. $63-64,72-81$.

5. Voir ma contribution dans Amish Quilts: Crafting an American Icon, Baltimore, 2013, p. 87-89. Sur les créateurs de quilts modernistes, voir Jonathan Gregory, " Early $20^{\text {th }}$ Century Ideas about Quilts and Art ", dans World Quilts: The American Story I International Quilt Study Center $\theta$ Museum, 2013, http://worldquilts.quiltstudy.org/americanstory/creativity/early20th-ideas-quilts-art (consulté le 30 novembre 2015). Voir aussi Virginia Stillinger, " From Attics, Sheds, and Secondhand Shops: Collecting Folk Art in America, 1880-1940 ", dans Virginia Tuttle Clayton, Elizabeth Stillinger, Erika Lee Doss éd., Drawing on America's Past: Folk Art, Modernism, and the Index of American Design, (cat. expo., Washington, D.C., National Gallery of Art, 2002), Washington, D.C., 2002, p. 52-53 ; Quilts in a Material World: Selections from the Winterthur Collection, Linda Eaton, (cat. expo., Winterthur, Winterthur Museum \& Country Estate, 2007), New York, 2007, p. 168-170.

6. Pour une analyse de ces débats, voir Elissa Auther, " Fiber Art and the Hierarchy of Art and Craft, 1960-80 ", dans The Journal of Modern Craft, 1, mars 2008, p. 13-33.

7. Michael James, « Beyond Tradition : The Art of the Studio Quilt ", dans American Craft, 45/1, mars 1985, p 16-22. Le "Studio Art Quilt " est un mouvement artistique né aux États-Unis au lendemain de la Seconde Guerre mondiale, et qui accorde la primauté aux modes d'expression artisanaux, dans la lignée du mouvement Arts and Crafts (N.D.L.T.).

8. " $[\ldots]$ subtle relationships between stripes and ground $[\ldots]$ carrying out a simple pattern " (cité dans Jean Lipman, Provocative Parallels: Nä̈ve Early Americans,
International Sophisticates, New York, 1975, p. 144).

9. " $[\ldots]$ seen out of their original context of use, hanging on a wall, they make it very plain how absurd the once jealously guarded hierarchical distinctions between 'folk' and 'high' art can be " (Robert Hughes, Amish: The Art of the Quilt, New York, 1990, p. 15).

10. "A quilt is an art object when it stands up like a man " (Susan E. Bernick, "A Quilt Is an Art Object When It Stands Up like a Man ", dans Cheryl B. Torsney, Judy Elsley éd., Quilt Culture: Tracing the Pattern, Columbia/ Londres, 1994, p. 134-150).

11. Patricia J. Cooper, Norma Bradley Allen, The Quilters: Women and Domestic Art, New York, 1977 ; Hedges, Silber, Ferrero, 1996, cité n. 4. Au sujet du genre et des quilts, voir Beverly Gordon, "Intimacy and Objects: A Proxemic Analysis of Gender-Based Response to the Material World ", dans Katherine Martinez, Kenneth L. Ames éd., The Material Culture of Gender: The Gender of Material Culture, Winterthur, 1997, p. 245-247.

12. Sur l'industrialisation et les quilts, voir Margaret $T$. Ordoñez, " Technology Reflected: Printed Textiles in Rhode Island Quilts ", dans Linda Welters, Margaret T. Ordoñez éd., Down by the Old Mill Stream: Quilts in Rhode Island, Kent, 2000, p. 134-146 ; Rachel Maines, " Paradigms of Scarcity and Abundance: The Quilt as an Artifact of the Industrial Revolution ", dans Jeannette Lasansky éd., In the Heart of Pennsylvania, Lewisburg, 1986, p. 84 ; Barbara Brackman, Clues in the Calico: A Guide to Identifying and Dating Antique Quilts, Charlottesville, 1989, p. 13. L'étude des documents de succession indiquant les motifs de quilts individuels inclue Sally Garoutte, "Early Colonial Quilts in a Bedding Context ", dans Uncoverings, 1, 1980, p. 18-27 ; Patricia J. Keller, " The Quilts of Lancaster County, Pennsylvania: Production, Context, and Meaning, 1750-1884 ", thèse, University of Delaware, 2007 ; Susan Margaret Prendergast, " Fabric Furnishings Used in Philadelphia Homes ", master, University of Delaware, 1977 ; Gloria Allen Seaman, "Bed Coverings, Kent County, Maryland, 1710-1820", dans Uncoverings, 6, 1985, p. 9-32. T. S. Arthur déplore la disparition des anciens ateliers de quilting, dans " The Quilting Party ", dans Godey's Lady's Book, septembre 1849, p. 185-186. Sur le sujet des quilts historiques, voir Barbara Brackman et al., "Quilting Myths and Nostalgia ", dans 'Workt by Hand': Hidden Labor and Historical Quilts, Catherine Morris éd., (cat. expo., Brooklyn, Brooklyn Museum, 2013), Brooklyn, 2012, p. 26-30.

13. Sur le Colonial Revival et l'art du quilt, voir Virginia Gunn, "Quilts for Milady's Boudoir ", dans Uncoverings, 10, 1989, p. 82 ; Virginia Gunn, "Perfecting the Past: Colonial Revival Quilts ", dans Marin F. Hanson, Patricia Cox Crews éd., American Quilts in the Modern Age, 1870-1940: The International Quilt Study Center Collections, Lincoln, 2009, p. 229-233 ; Beverly Gordon, "Spinning Wheels, Samplers, and the Modern Priscilla: The Images and Paradoxes of Colonial Revival Needlework ", dans Winterthur Portfolio, 33, 2/3, été-automne 1998, p. 163-194.

14. Marin Hanson, "Modern, Yet Anti-Modern: Two Sides of Late-Nineteenth- and Early -Twentieth-Century Quiltmaking ", dans Uncoverings, 29, 2008, p. 105-136. 
15. Eleanor Levie, American Quiltmaking: 1970-2000, Paducah, 2004, p. 50-66; Christine Humphery, "Bigger, Faster, Better, More ", dans World Quilts: The American Story I International Quilt Study Center $\theta$ Museum, 2013, http:// worldquilts.quiltstudy.org/americanstory/business/biggermore (consulté le 30 novembre). Sur l'internationalisation des quilts de style américain, voir Nao Nomura, "The Development of Quiltmaking in Japan since the 1970s ", dans Uncoverings, 31, 2010, p. 105-130.

16. Marie D. Webster, Quilts, Their Story and How to Make Them, Garden City, 1915, p. 80.

17. "indisputably women's art " (Patricia Mainardi, "Quilts, The Great American Art ", dans The Feminist Art Journal, 2/1, 1973, p. 1).

18. Elissa Auther, "A Brief History of Quilts in Contemporary Art ", dans Roderick Kiracofe éd., Unconventional $\theta$ Unexpected: American Quilts below the Radar, 1950-2000, New York, 2014, p. 110-111 ; Janet Catherine Berlo, "'Acts of Pride, Desperation, and Necessity': Aesthetics, Social History, and American Quilts ", dans Janet Catherine Berlo, Patricia Cox Crews éd., Wild by Design: Two Hundred Years of Innovation and Artistry in American Quilts, Lincoln/Seattle, 2003, p. 6-7 ; Auther, 2008, cité n. 6.

19. Maines, 1986, cité n. 12, p. 84 .

20. Robert Shaw, American Quilts: The Democratic Art, 1780 2007, New York, 2009, p. 1.

21. Merikay Waldvogel, Deborah Rake, Marin F. Hanson, "Repackaging Tradition: Pattern and Kit Quilts ", dans Hanson, Cox Crews, 2009, cité n. 13, p. 306-311.

22. Jennifer F. Goldsborough, "An Album of Baltimore Album Quilt Studies ", dans Uncoverings, 15, 1994, p. 73-110; pour des images, voir le site des Daughters of the American Revolution, "Albums ", dans Eye on Elegance, 2014, http://eyeonelegance.dar.org/exhibition/ albums-quilts (consulté le 30 novembre 2015).

23. Sur l'origine de ceci, et d'autres mythes concernant les quilts, voir Virginia Gunn, "From Myth to Maturity: The Evolution of Quilt Scholarship ", dans Uncoverings, 13, 1992, p. 195-196.

24. Maines, 1986, cité n. 12, p. 86 .

25. " [...] every scrap and remnant of woolen material left from the manufacture of garments was saved. To supplement these, the best parts of worn-out garments were carefully cut out, and made into quilt pieces " (Webster, 1915, cité n. 16, p. 80).

26. "In mansion house and frontier cabin, from the Cavaliers of Jamestown to the Puritans of Plymouth, scraps of linen, cotton, silk and wool were jealously saved and pieced into patchwork quilts " (Ruth E. Finley, old Patchwork Quilts and the Women Who Made Them, Philadelphie/Londres, 1929, p. 21).

27. The Quilts of Gee's Bend: Masterpieces from a Lost Place, William Arnett et al., (cat. expo., Houston, Museum of Fine Arts, 2002), Atlanta, 2002 ; voir Alicia Carroll, "The Quilts of Gee's Bend in Context ", site de l'Auburn University, http://www.auburn.edu/academic/other/geesbend/home.html (consulté le 30 novembre 2015).
28. Kiracofe, 2014, cité n. 18.

29. Lucinda Ward Honstain, Reconciliation Quilt, Lincoln, 1867 ; voir aussi Rebecca Onion, « A Brooklyn Woman's Colorful Quilt, Illustrating Her Experience of the Civil War ", dans The Vault, Slate, 18 mars 2014, www.slate. com/blogs/the_vault/2014/03/18/reconciliation_quilt_lucinda_ward_honstain_s_vision_of_the_civil_war_in.html (consulté le 30 novembre 2015).

30. Kyra E. Hicks, This I Accomplish: Harriet Powers' Bible Quilt and Other Pieces: Quilt Histories, Exhibition Lists, Annotated Bibliography and Timeline of a Great African American Quilter, 2009 ; National Museum of American History, "1885-1886 Harriet Powers's Bible Quilt ", http://americanhistory. si.edu/collections/search/object/nmah_556462 (consulté le 30 novembre 2015) ; Museum of Fine Arts, Boston, " Pictorial Quilt ", www.mfa.org/collections/object/pictorial-quilt-116166 (consulté le 30 novembre 2015) .

31. Merikay Waldvogel, Patchwork Souvenirs of the 1933 World's Fair, Nashville, 1993 ; Emma Mae Leonhard, From 1833 to 1933, vers 1933, Lincoln, University of NebraskaLincoln, International Quilt Study Center \& Museum.

32. Voir Arthur, 1849, cité n. 12 ; Christine Humphery, "The Quilting Bee?", dans World Quilts: The American Story I International Quilt Study Center $\theta$ Museum, 2013, http://worldquilts.quiltstudy.org/americanstory/creativity/ quiltingbee (consulté le 30 novembre) ; Laurel Horton, "An 'Old-Fashioned Quilting' in 1910 ", dans Uncoverings, 21, 2000, p. 1-26 ; Ricky Clark, "Sisters, Saints, and Sewing Societies: Quiltmakers' Communities ", dans Quilts in Community: Ohio's Traditions, Nashville, 1991 ; Marsha L. MacDowell, "North American Indian and Native Hawaiian Quiltmaking ", dans To Honor and Comfort: Native Quilting Traditions, Marsha MacDowell, C. Kurt Dewhurst éd., (cat. expo., East Lansing, Michigan State University Museum, 1997), Santa Fe, 1997, p. 61-67, http://museum. msu.edu/museum/tes/thc/exhibit\%201.htm (consulté le 30 novembre) ; Nancy Callahan, The Freedom Quilting Bee, Tuscaloosa, 1987 ; Smithsonian Folklife Festival, "The Quilting Bee ", dans Creativity and Crisis: Unfolding the AIDS Memorial Quilt, 2012, http://www.festival.si.edu/past-festivals/2012/Creativity_and_Crisis/quilting_bee/index.aspx (consulté le 30 novembre).

33. Linda Otto Lipsett, Remember Me: Women $\theta$ Their Friendship Quilts, San Francisco, 1985 ; Smucker, 2013, cité n. 5, p. 34-42 ; C. Kurt Dewhurst, Marsha L. MacDowell, "Stars of Honor: The Basketball Star Quilt Ceremony ", dans To Honor and Comfort, 1997, cité n. 32, p. 129-135.

34. Pour une étude approfondie du quilt de Ricard, voir Beverly Gordon, "My Crazy Dream ", dans Hanson, Cox Crews, 2009, cité n. 13, p. 134-137 ; voir aussi la discussion et les images du quilt de Ricard dans Marin F. Hanson, "Quilts in Context ", dans World Quilts: The American Story I International Quilt Study Center $\theta$ Museum, 2013, http://worldquilts.quiltstudy.org/americanstory/ quiltsare/quiltsincontext (consulté le 30 novembre2015).

Janneken Smucker, West Chester, University of Pennsylvania

JSmucker@wcupa.edu 